\title{
Characterization of density fluctuations during ELMs in the DIII-D tokamak
}

\author{
S. Coda ${ }^{\mathrm{a}, *}$, M. Porkolab ${ }^{\mathrm{a}}$, K.H. Burrell ${ }^{\mathrm{b}}$ \\ ${ }^{a}$ Department of Physics and Plasma Science and Fusion Center, \\ Massachusetts Institute of Technology, Cambridge, Massachusetts \\ ${ }^{\mathrm{b}}$ General Atomics, San Diego, California \\ United States of America
}

\begin{abstract}
Bursts of turbulence associated with ELMs have been studied systematically in DIII-D with a multichannel phase contrast imaging (PCI) diagnostic, which is sensitive to the long poloidal wavelength components of the density fluctuations in the outer edge of the tokamak. A comparison of the temporal dynamics of the turbulence with the signature $\mathrm{D}_{\alpha}$ signal from the divertor has revealed systematic differences between type I and type III ELMs: even though precursor fluctuations are sometimes seen before type I ELMs, the PCI signal level remains high until the peak in the $\mathrm{D}_{\alpha}$ signal; by contrast, in type III ELMs the fluctuation burst precedes the $\mathrm{D}_{\alpha}$ peak by $0.4-0.6 \mathrm{~ms}$. Type I ELMs can generate 'echoes', i.e. secondary bursts, in the scrape-off layer. Coherent modes are observed during type III ELMs only. The radial and temporal correlation structures and the spectral properties of the turbulence during the transient ELM phase have been reconstructed by averaging over multiple ELMs, in order to improve the statistical accuracy. ELM turbulence is found to share many properties with L mode turbulence, including the main qualitative features of radial wavenumber and frequency spectra and radial dispersion relations. However, features unique to ELM turbulence are also identified.
\end{abstract}

\section{Introduction}

ELMs [1] are semi-periodic tokamak edge instabilities that occur in $\mathrm{H}$ mode and transiently destroy the transport barrier for the brief duration of the mode $[2,3]$. The average diffusion rate during an ELMing $\mathrm{H}$ mode is higher than that during an ELMfree $\mathrm{H}$ mode; on the other hand, ELMs serve the useful purpose of limiting the density buildup and of cleansing the plasma from impurities that accumulate during the enhanced confinement stage. For these reasons, ELMs are considered essential for reactor operation. However, ELMs can also place very large transient power loads on the tokamak wall $[4,5]$. The need to control the frequency and amplitude of ELMs in a future reactor motivates the focus of much current research on this phenomenon.

MHD instabilities are believed to trigger these modes [6], which are characterized by levels of turbulence and confinement similar to those of L mode. Specifically, type I (or 'giant') ELMs have been linked to ideal modes (ballooning and possibly low toroidal number kink modes) [7-9], while type III ELMs are thought to be related to resistive MHD

* Present and permanent affiliation: Centre de Recherches en Physique des Plasmas, Ecole Polytechnique Fédérale de Lausanne, Lausanne, Switzerland. instabilities [10]. Type II ('grassy') ELMs are observed in strongly shaped plasmas at high triangularity [11], when the magnetic shear is in the connecting region between the first and second regions of stability to ballooning modes. While several theoretical models have been proposed to explain this phenomenology [6], much remains unknown about the detailed physical mechanisms and dynamics of ELMs. Thus, accumulation of experimental information is still of crucial importance.

Coherent or semi-coherent precursor fluctuations have often been seen in the $20-100 \mathrm{kHz}$ spectral region, from 1 up to $20 \mathrm{~ms}$ before an ELM, both in density (by reflectometry [12]) and in magnetic field $[10,13,14]$. Broadband precursors to type I ELMs have also been documented [12]. Less attention has been devoted to fluctuations occurring during the ELM itself, partly because of the short duration of the event. It is known that the ELM is mainly a turbulent phenomenon and that the level of turbulence is high compared with that of ELM-free $\mathrm{H}$ mode $[10,12]$. In this article we examine the characteristics of density fluctuations for the different classes of ELM in DIII-D. The diagnostic employed in this study is a phase contrast imaging (PCI) system, which measures the line integrals of the fluctuations at the outer edge of the tokamak over several radially spaced vertical chords. 


\section{S. Coda et al.}

Two major systematic differences between type I and type III ELMs have been identified in this study: firstly, the fluctuation amplitude is high at the time of maximum diffusivity for a type I ELM, whereas for a type III ELM the fluctuation burst precedes the $\mathrm{D}_{\alpha}$ peak by $0.4-0.6 \mathrm{~ms}$; this could be related to ideal and resistive MHD times, respectively. Secondly, type III ELMs are sometimes also accompanied by a coherent, outward propagating mode at approximately $100 \mathrm{kHz}$, which may be related to a resistive MHD instability. In some cases in which measurements have been extended deep into the SOL, 'echoes', i.e. additional fluctuation bursts, have been observed there after type I ELMs.

A technique of compound averaging over multiple ELMs was used for correlation and spectral analysis to improve the statistical signal to noise ratio. This technique permits the reconstruction of the frequency and radial wavenumber spectra of the turbulence at long poloidal wavelengths. The turbulent spectra and dispersion relations during ELMs are generally similar to those of L mode [15], but the absolute amplitude of the turbulence is larger in the case of ELMs.

The remainder of this article is organized as follows. In Section 2 we shall describe the diagnostic technique and the experimental apparatus, as well as the interpretation of the data. Section 3 introduces the data analysis techniques used in this study. The experimental results are presented in Section 4, followed by a discussion and conclusions in Section 5 .

\section{Experimental technique and set-up}

The PCI diagnostic $[16,17]$ employs a $20 \mathrm{~W} \mathrm{CO}_{2}$ laser beam of $10.6 \mu \mathrm{m}$ wavelength and $7.6 \mathrm{~cm}$ width, which is launched vertically through the outer edge of the DIII-D plasma (Fig. 1), and is then passed through a Zernike phase contrast filter [18] to create a horizontal image of the plasma onto a 16 element, $\mathrm{LN}_{2}$ cooled, $\mathrm{HgMnTe}$ linear photovoltaic detector array. The wavenumber transfer function is real valued and essentially constant down to a cut-off value, below which it rapidly falls to zero [16]; the 1 / e point is $0.65 \mathrm{~cm}^{-1}$. These properties ensure that the system produces a spatial image of the fluctuating component of the density. Thus, each of the 16 detector elements measures the vertical line integral of the density fluctuations at one specific major radius within the width of the beam; the 16 measurements correspond to 16 equally spaced horizontal locations, typically distributed from just inside to just outside

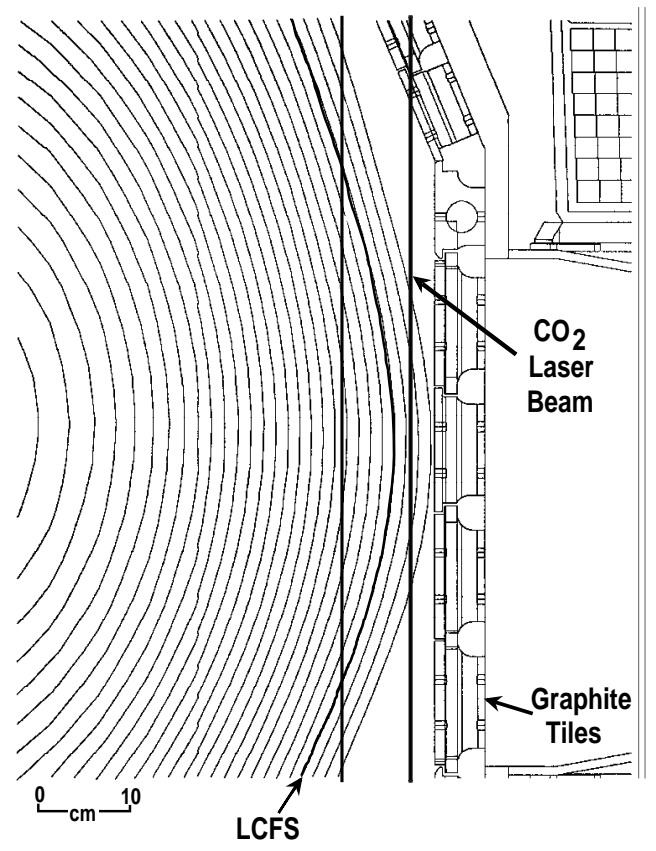

Figure 1. Geometry of the PCI measurement. The outer portions of the magnetic flux surfaces of a typical DIII-D plasma are shown. The position of the LCFS can vary from discharge to discharge.

the LCFS. Since the beam is fixed, the location relative to the LCFS depends on the positioning of the latter; the range $0.80<\psi_{n}<1.17$ has been explored in the course of this study, $\psi_{n}$ being the poloidal flux normalized to its value at the LCFS. In the standard configuration, the outermost chords sample fluctuations originating only in the SOL, while the innermost ones extend to $2-3 \mathrm{~cm}$ inside the LCFS. Thus, the $\mathrm{PCI}$ beam also encompasses the $\mathrm{H}$ mode transport barrier, which is approximately $1-2 \mathrm{~cm}$ wide and is located just inside the LCFS [19]. Only eight signals, freely chosen from the 16 , can be collected in a given plasma discharge.

The radial separation between the chords can be varied, but is typically set at $0.5 \mathrm{~cm}$. Anti-aliasing spatial optical filtering restricts the wavenumber spectrum to $k<6 \mathrm{~cm}^{-1}$. The accessible frequency range is $8-1000 \mathrm{kHz}$, the upper limit being set by the acquisition electronics and the lower limit by mechanical vibrations. The minimum detectable density is approximately $10^{15} \mathrm{~m}^{-3}$; for typical fluctuation levels, the signal to noise ratio is in the range 10-100. An absolute calibration can be performed by means of acoustic waves in air. An accuracy of only about $30-40 \%$ can be attained in the absolute amplitude, but the relative amplitude from chord to chord is known to an accuracy of about $10-15 \%$. A relative 
calibration is carried out in each plasma discharge, through a short acoustic pulse launched $50 \mathrm{~ms}$ before the shot.

The vertical line integration engenders a selection rule for the measured wave vectors: since any component along the direction of integration will be averaged out, the measured wave vectors must be purely horizontal. Because the toroidal component of the wave vector is well known to be negligibly small [20], this selection rule implies that, on the midplane, the measured wave vector is purely radial $\left(k_{r}\right)$. Away from the midplane the poloidal projection $k_{\theta}$ becomes non-zero as a result of field line curvature, but remains small because of the edge location [21] (Fig. 1). The selection rule can thus be written $\left|k_{r}\right| \gg\left|k_{\theta}\right|$, and has been confirmed by a numerical calculation of the responsivity of the system in $\left(k_{r}, k_{\theta}\right)$ space for typical DIII-D discharges [22]. This implies that, in spite of the large $\boldsymbol{E} \times \boldsymbol{B}$ poloidal and toroidal flows experienced by the plasma in response to the radial electric field, especially in $\mathrm{H}$ mode, the PCI diagnostic is subject to negligible Doppler shifts. This is confirmed by the experimental frequency spectra, which are typically monotonically decreasing and show little or no activity above about $150 \mathrm{kHz}$ [21]. Thus, turbulence is studied essentially in the plasma frame of reference. A further implication is that the spectrum derived from chord to chord correlation analysis closely approximates the radial spectrum $\left.S\left(k_{r}\right)\right|_{k_{\theta} \sim 0, k_{\phi} \sim 0}$. This has also been verified by modelling with a variety of turbulence spectra, numerically line integrated in the real DIIID geometry [17, 21, 23].

\section{Data analysis techniques}

Data analysis consists primarily of a series of calculations of standard statistical functions: these include the RMS values, the autospectral and crossspectral density functions in the frequency domain, and the autocorrelation and cross-correlation functions in the time domain. Digital high pass filtering is always applied at a cut-off frequency of $8 \mathrm{kHz}$ to eliminate vibrational effects. When calculating frequency spectra, smoothing must be performed over a finite frequency interval: therefore in the spectral plots shown in this article the lower frequency bound exceeds $8 \mathrm{kHz}$ by one half the width of the averaging interval.

The assumptions of stationarity and ergodicity are used to replace ensemble averaging with time averaging [24], and care is always taken to effect the averaging over intervals in which no drastic variations in the physical conditions are observed. Data are also preconditioned before all FFTs by linear trend removal. The evolution of the statistical functions themselves can then be studied over longer timescales.

Higher level data reduction is employed to study the structure of spatial correlation functions and spectra. For each frequency, the complex coherence function (the normalized cross-spectral function) can be calculated as a function of the radial separation $\Delta R$. It has been found empirically that this function conforms rather well to the form

$$
\begin{aligned}
\gamma(\Delta R ; f)= & \exp \left(-\frac{(\Delta R)^{2}}{L_{c R}^{2}}\right) \\
& \times\left(\frac{1+P_{R}}{2} e^{i k_{0, R} \Delta R}+\frac{1-P_{R}}{2} e^{-i k_{0, R} \Delta R}\right)
\end{aligned}
$$

where $k_{0, R}$ and $L_{c R}$ bear a natural interpretation as the average radial wavenumber and correlation length, respectively, and $P_{R}$ as a propagation coefficient ( 1 for purely outward, -1 for purely inward propagation). All these parameters are functions of the frequency. In particular, this fitting procedure permits the determination of an approximate dispersion relation $k_{0, R}(f)$. A minimum $\chi^{2}$ algorithm is used in this fit, allowing for error bars on both the independent variable (the spatial uncertainty) and the dependent variable (the statistical uncertainty), which are propagated to the calculated fit parameters.

The spatial Fourier transform of the unnormalized cross-spectral function, divided by the instrumental PCI transfer function, gives the complete spectrum of the fluctuations, $S\left(k_{R}, f\right)$. Several techniques for sparse Fourier transforms have been compared, yielding acceptably similar results; a maximum entropy algebraic reconstruction technique algorithm [25] is employed preferentially.

A basic difficulty in any statistical analysis of ELM behaviour is the short duration of the phenomenon, which results typically in poor statistics. In order to increase the number of statistical samples, a multiple interval averaging technique has been adopted for this study; this technique entails carrying out the averages over intervals composed of several discontinuous segments, each of which lies within the lifetime of an ELM. Under the assumption that individual ELMs are characterized by similar spectral properties, this technique increases the number 
of samples by a factor equal to the number of segments used in the analysis. Note that the assumed property is not strictly stationarity [24], since the RMS value of the fluctuations is found in fact to vary significantly from ELM to ELM; only stationarity of the correlation properties of the signals is invoked. In all cases, the segments were selected carefully by restricting each estimate to only one class of ELMs and to a single discharge, and by excluding all ELMs which could be considered outliers from the specific point of view of their density fluctuation content (e.g., ELMs that were too low in amplitude to contribute to the statistics, or the 'echo' ELMs described in the next section). This technique proved inapplicable to grassy ELMs, owing to their very short duration and complex dynamics, which will be briefly illustrated in the next section.

\section{Experimental results}

We employed the PCI diagnostic to perform a systematic study of turbulence in the type I and type III ELMs that occur in the $\mathrm{H}$ mode plasmas of DIII-D. A more qualitative description of grassy ELMs and of the so-called X event that often terminates the $\mathrm{VH}$ mode phase of the discharge will also be given in this section.

\subsection{ELM dynamics}

We shall discuss here the time evolution of the ELM event and the temporal correlations between the increase in the $\mathrm{D}_{\alpha}$ emission signal, which identifies the ELM experimentally, and the fluctuation enhancement.

A comparison between the PCI RMS signal and the $\mathrm{D}_{\alpha}$ signal for a series of type I ELMs, and for two ELMs in detail, is shown in Fig. 2. The detail also shows the evolution of the PCI autopower spectrum, at a location $0.5 \mathrm{~cm}$ inside the LCFS, in contour form. For each ELM, the fluctuation level often begins to rise dramatically up to $2-3 \mathrm{~ms}$ before any change is observed in the emission signal; this 'precursor' turbulence is generally observed more prominently further inside the plasma than near the boundary, but has no apparent regularity in time: as clearly demonstrated by Fig. 2, precursors may appear before an ELM and not before the next one. Within the resolution of the measurement, as documented in the figure, the peak in the $\mathrm{D}_{\alpha}$ signal occurs before the fluctuation signal has begun its descent. However, the $\mathrm{D}_{\alpha}$ signal invariably decays more

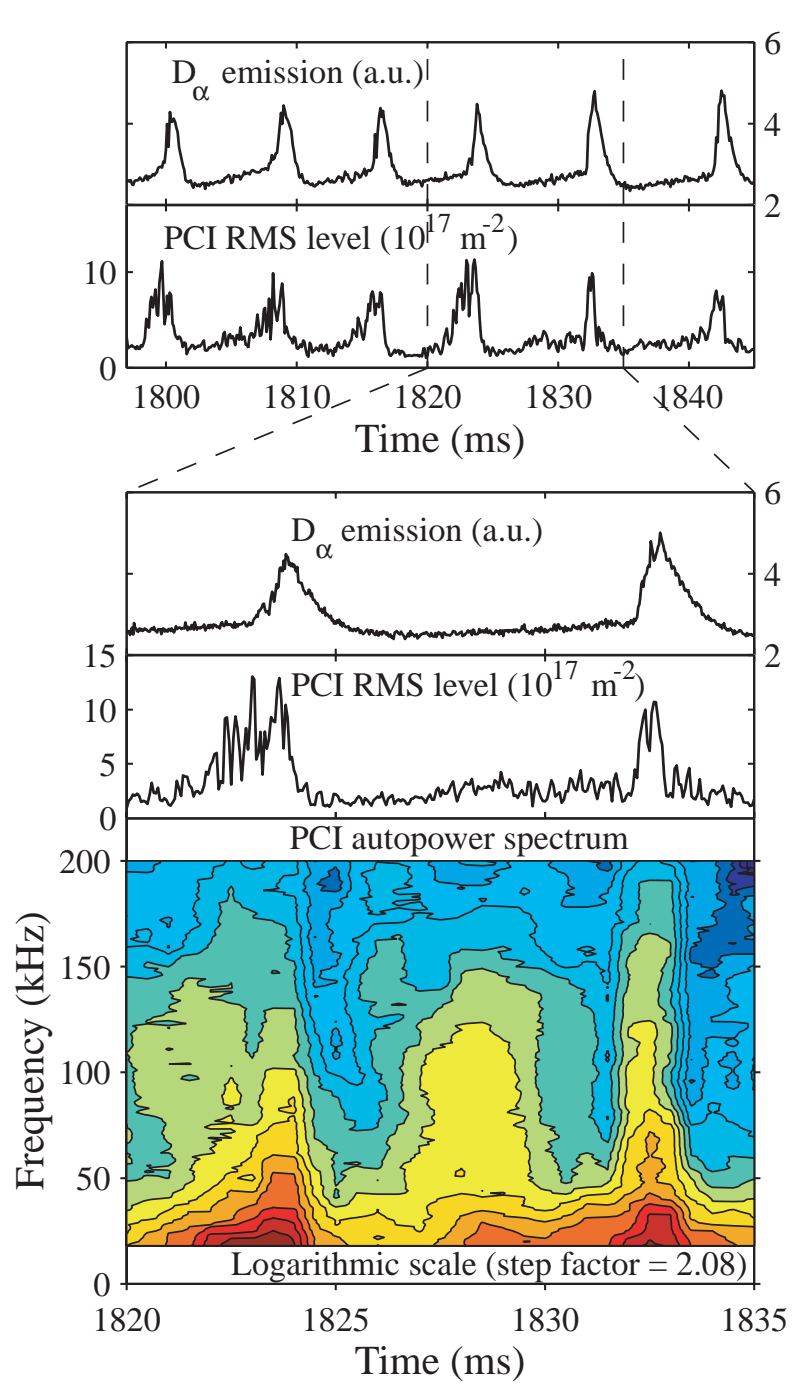

Figure 2. Top: time traces of the $\mathrm{D}_{\alpha}$ line emission from the divertor and of the PCI RMS level; bottom: enlargement of the same traces for two type I ELMs, and a contour plot of the time evolution of the PCI autopower spectrum (smoothing interval, $20 \mathrm{kHz}$; values increase from blue to red). The PCI chord intersects the midplane $0.5 \mathrm{~cm}$ inside the LCFS. The statistical uncertainties are $10 \%$ on the RMS level and $22 \%$ on the autospectrum; the absolute amplitude calibration error is $40 \%$. Plasma parameters: $B_{T}=2.1 \mathrm{~T}, I_{p}=1.25 \mathrm{MA}$, $\bar{n}_{e}=3.5 \times 10^{19} \mathrm{~m}^{-3}$ (discharge 80041$)$.

gradually than the fluctuation signal (inside the confinement region). A slower rise in the turbulence level is seen in the enlargement section of Fig. 2, starting soon after the first ELM and leading to the second one; this activity, however, always appears to match a corresponding gradual rise in the $\mathrm{D}_{\alpha}$ signal. The time evolution is essentially the same on all the 


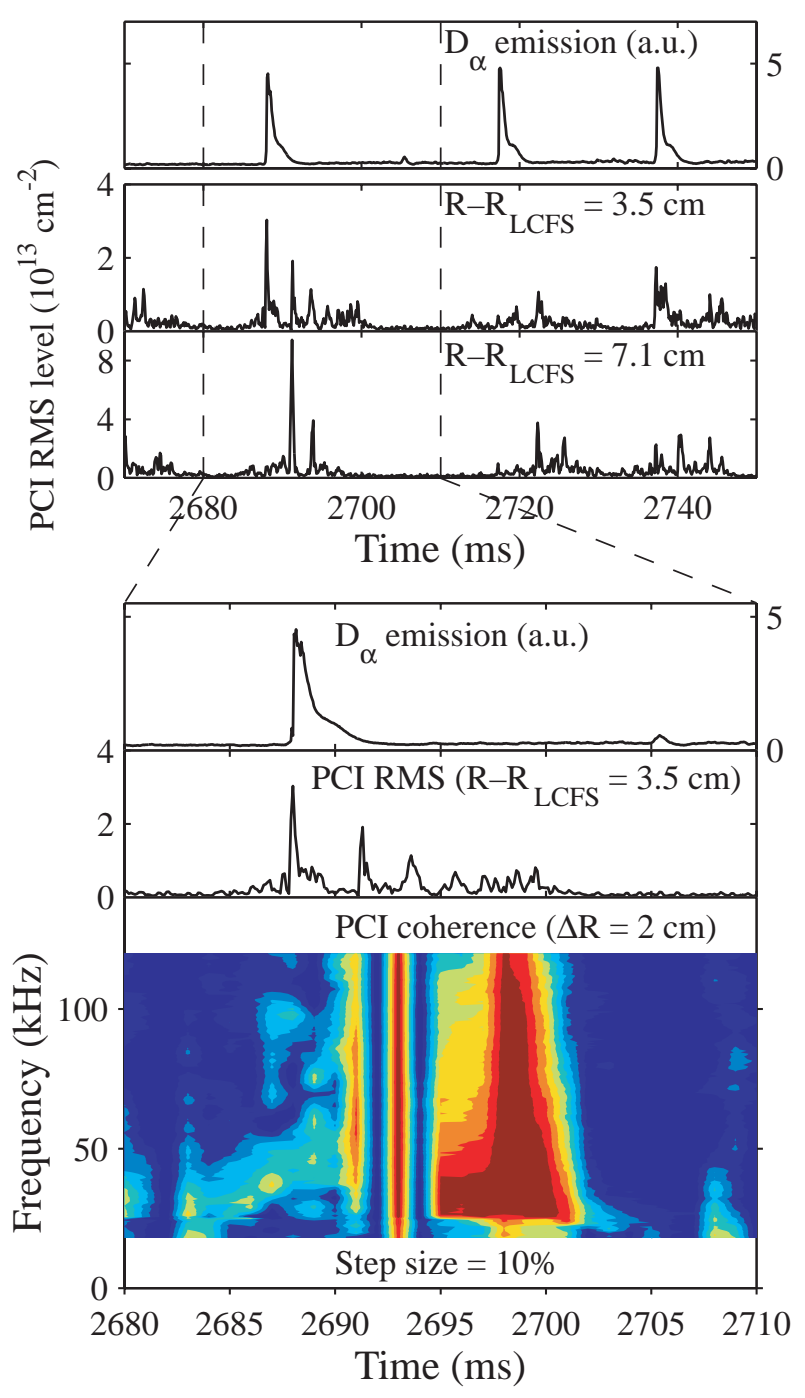

Figure 3. Top: time traces of the $\mathrm{D}_{\alpha}$ line emission from the divertor and of the PCI RMS level at two different SOL locations, 3.5 and $7.1 \mathrm{~cm}$ outside the LCFS; bottom: enlargement of one type I ELM with a contour plot of the time evolution of the coherence between two PCI chords $2 \mathrm{~cm}$ apart, with the middle point located $3.5 \mathrm{~cm}$ outside the LCFS (smoothing interval, $20 \mathrm{kHz}$ ). The statistical uncertainties are $7 \%$ on the RMS level and $12 \%$ on the coherence. Plasma parameters: $B_{T}=2.1 \mathrm{~T}$, $I_{p}=1.4 \mathrm{MA}, \bar{n}_{e}=8 \times 10^{19} \mathrm{~m}^{-3}$ (discharge 75931$)$.

spatial channels of the PCI diagnostic, with no measurable delay between channels, whether inside or outside the LCFS. The frequency spectrum is broadband and monotonically decreasing.

PCI measurements of type I ELMs deep in the SOL, an example of which is shown in Fig. 3, reveal a somewhat different phenomenology from that of the main plasma. Each ELM is followed by a series of



Figure 4. Top: time traces of the $\mathrm{D}_{\alpha}$ line emission from the divertor and of the PCI RMS level; bottom: enlargement of the same traces for one type III ELM, and a contour plot of the time evolution of the PCI autopower spectrum (for a smoothing interval of $20 \mathrm{kHz}$ ). The PCI chord intersects the midplane $1.7 \mathrm{~cm}$ inside the LCFS. The statistical uncertainties are $10 \%$ on the RMS level and $22 \%$ on the autospectrum. Plasma parameters: $B_{T}=2.1 \mathrm{~T}, I_{p}=1.0 \mathrm{MA}, \bar{n}_{e}=5.5 \times 10^{19} \mathrm{~m}^{-3}($ discharge 74455$)$.

'echoes', in the form of additional fluctuation bursts; these echoes vary in magnitude from ELM to ELM, and from chord to chord for each ELM, seemingly at random. In addition, the amplitude of the bursts may exceed that of the main ELM. Since these echoes appear to be very short lived, it may be speculated that this variability is due in part to the finite sampling rate: i.e. the fluctuation peak may fall between 
(a) PCI autopower spectrum

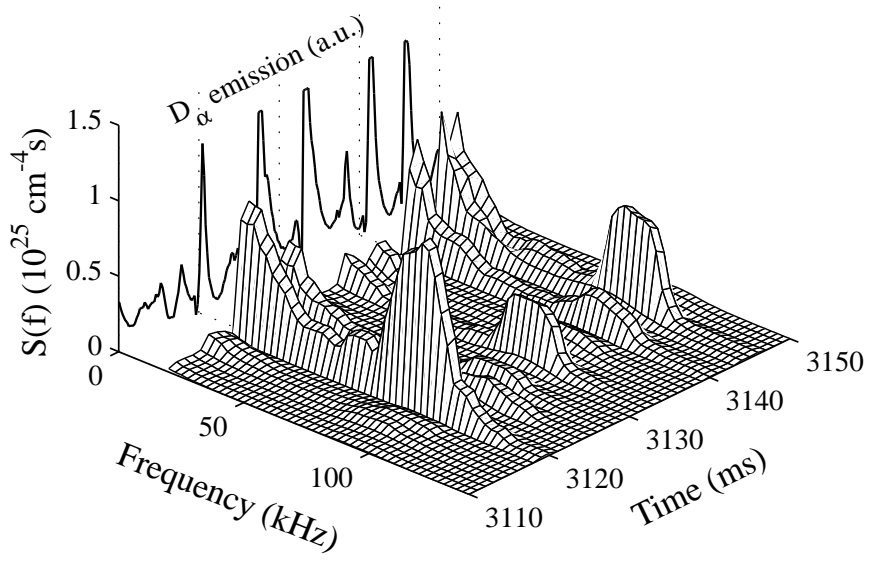

(b) PCI coherence and cross-phase $(96 \mathrm{kHz}, 1 \mathrm{ELM})$

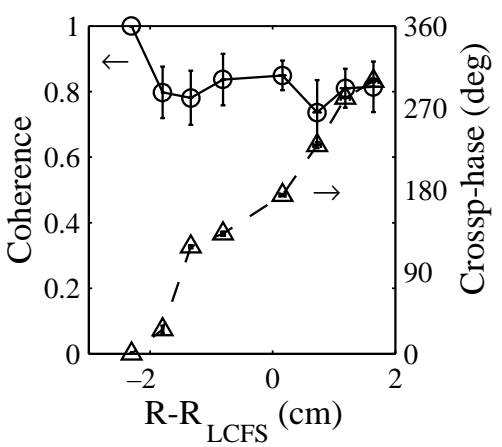

Figure 5. (a) Time evolution of the PCI autopower spectrum $1.7 \mathrm{~cm}$ inside the LCFS (estimated over a $20 \mathrm{kHz}$ smoothing interval, with a statistical uncertainty of $16 \%$ ), and a time trace of the $\mathrm{D}_{\alpha}$ line emission from the divertor; (b) coherence (circles) and cross-phase (triangles) at $96 \mathrm{kHz}$, averaged from 3119 to $3121 \mathrm{~ms}$ and smoothed over $10 \mathrm{kHz}$, with respect to a chord located $2.3 \mathrm{~cm}$ inside the LCFS, plotted as a function of the distance from the LCFS (with maximum statistical errors of, respectively, $11 \%$ and $8^{\circ}$ ). The positive slope in the phase function indicates outward propagation. The discharge is the same as that for Fig. 4.

two samples in some cases, and its amplitude would then be underestimated.

It should be noted that the measurement relative to Fig. 3 extends to $7.1 \mathrm{~m}$ outside the LCFS; the level of turbulence associated with the ELM there is extremely high in comparison with any activity observed in L mode. A peculiar feature of type I ELMs in the SOL is that the correlation level of the fluctuations is typically larger in the echo phase than during the ELM itself, as shown at the bottom of Fig. 3 for one specific pair of chords. Precursors are also sometimes seen in the SOL but are, as a rule, of more modest amplitude than those inside the LCFS.

An examination of the dynamics of type III ELMs leads to the establishment of a clear experimental distinction between type III and type I ELMs. As shown in the comparison of the $\mathrm{D}_{\alpha}$ emission signal and the PCI RMS signal in Fig. 4, in the case of a type III ELM the peak in the fluctuation amplitude occurs well in advance of the peak in the $\mathrm{D}_{\alpha}$ signal, and when the latter occurs the fluctuation level is already decreasing or has reached the baseline $\mathrm{H}$ mode level. The delay is always between 0.4 and $0.6 \mathrm{~ms}$. The evolution of the autopower spectrum shows a monotonically decreasing spectrum similar to that of type I ELMs, but with the addition of a high frequency feature at $85-105 \mathrm{kHz}$.

The turbulent activity associated with type III ELMs does not exhibit any significant spatial variation, both inside the LCFS and in the SOL. As with type I ELMs, no measurable delay is observed across the width of the PCI beam.

A different view of the evolution of the autopower spectrum in time is provided by Fig. 5(a), which depicts five type III ELM events in succession. Both the broadband turbulent activity and the high frequency feature are clearly associated with individual ELMs, and in fact precede them as stated before.

Let us now focus on the high frequency feature. Figure 5(b) shows that the radial coherence, calculated at the peak frequency of $96 \mathrm{kHz}$, is close to unity across the PCI range from inside to outside the LCFS. Hence this is in fact a coherent mode, even though the autopower plot could not reveal its coherent nature because of the smoothing required by the autopower estimation. Moreover, the cross-phase increases with radial separation, and is consistent with a coherent outward propagating mode with a wavelength of approximately $4.5 \mathrm{~cm}$ (thus a phase velocity of $\approx 43 \mathrm{~km} / \mathrm{s}$ ). However, in the case of coherent modes, a unique reconstruction of the mode structure from the measured line integrated signals is not possible. A long wavelength poloidally propagating mode (poloidal mode number $10 \lesssim m \lesssim 20$ ) with a strong up-down asymmetry would also be compatible with the observed wave pattern. (A poloidally propagating mode is 'seen' by the diagnostic as inward moving on one side of the 




Figure 6. Top, time trace of the $\mathrm{D}_{\alpha}$ line emission from the divertor, showing an $\mathrm{L}-\mathrm{H}$ transition and grassy ELMs; middle, enlargement of the same trace and the PCI RMS fluctuation level; bottom, further enlargement of both traces. The PCI chord intersects the midplane $1.4 \mathrm{~cm}$ inside the LCFS. The statistical uncertainty on the RMS level is $10 \%$. Plasma parameters: $B_{T}=2.1 \mathrm{~T}$, $I_{p}=1.35 \mathrm{MA}, \bar{n}_{e}=4 \times 10^{19} \mathrm{~m}^{-3}$ (discharge 78863).

midplane and outward moving on the other: thus an up-down symmetric mode would result in an apparent standing wave pattern.)

The same result is found for all type III ELMs that contain this mode, although the frequency varies by as much as $\pm 10 \mathrm{kHz}$. However, not all type III ELMs exhibit this mode, and no correlations were found between its onset and any other measurements. In particular, no similar coherent signal is seen in any of the signals from the soft $\mathrm{X}$ ray diode array; by contrast, normal, non-bursty MHD activity is usually detected by both PCI and the soft X ray diodes.

Type II, or grassy, ELMs present qualitatively similar features to the other two types of ELM from the point of view of edge turbulence; i.e. ELM activity is accompanied by an increased and oscillating fluctuation amplitude, as shown in Fig. 6 .
However, the short duration and high repetition rate of grassy ELMs have hampered all efforts to carry out a meaningful statistical analysis with PCI thus far. As shown in the bottom part of Fig. 6, the periods of the ELMs on the $\mathrm{D}_{\alpha}$ and PCI signals appear similar; peaks in one signal appear to be correlated with valleys in the other, and vice versa. The continuous transition from one ELM to the next, however, makes it difficult to establish whether this is the result of a true anticorrelation or of a delay. Moreover, these observations are only qualitative and are accompanied by sufficient irregularities that a temporal correlation analysis of the two signals has produced no clear results.

Figure 7 shows an example of an $\mathrm{X}$ event signalling the termination of the $\mathrm{VH}$ mode of a discharge. The $\mathrm{X}$ event [26] appears as an abnormally large type I ELM on the $\mathrm{D}_{\alpha}$ signal, and is generally followed by regular ELMing activity. This description also applies to the fluctuation measurements, as seen both in the PCI RMS signal and in the PCI autopower spectrum, which are consistent with normal type I ELM activity.

\subsection{Frequency spectra}

Typical autospectra for type I and type III ELMs are shown in Fig. 8. The spectrum of type I ELMs is compared with an L mode spectrum for the same discharge; no L mode data were available for comparison with the somewhat more elusive type III ELMs. The spectral content of type I ELMs is similar to that of L mode turbulence [15], and the two spectra obey similar inverse square laws (between $f^{-1}$ and $\left.f^{-3}\right)$ : this similarity was confirmed in all the cases examined. The ratio of the absolute values of the spectra, on the other hand, varies considerably from shot to shot, from a factor of 1 to a factor of 20 inside the LCFS. In no case was the ELM autospectral power smaller than its L mode counterpart; the value of about 10, seen in Fig. 8(a), can be considered typical.

The spectrum of type III ELMs has a broadband component, which is similar in shape and magnitude (for comparable plasma conditions and spatial locations) to the type I ELM spectrum, and the narrowband component centred at $96 \mathrm{kHz}$, which is in fact coherent as was discussed before. In general, the appearance of one or more narrowband components could be indicative of spectral features that vary from ELM to ELM and thus violate the stationarity assumption. In such a case, a verification 




Figure 7. Top, time traces of the $\mathrm{D}_{\alpha}$ line emission from the divertor and of the PCI RMS level; bottom, enlargement of the same traces for an $\mathrm{X}$ event and a contour plot of the time evolution of the PCI autopower spectrum (for a smoothing interval of $20 \mathrm{kHz}$ ). The PCI chord intersects the midplane $1.2 \mathrm{~cm}$ inside the LCFS. The statistical uncertainties are $10 \%$ on the RMS level and $22 \%$ on the autospectrum. Plasma parameters: $B_{T}=2.16 \mathrm{~T}$, $I_{p}=2.0 \mathrm{MA}, \bar{n}_{e}=9 \times 10^{19} \mathrm{~m}^{-3}$ (discharge 79633)

of the spectra of individual ELMs is essential. The feature shown here does vary considerably in amplitude from ELM to ELM, but its frequency fluctuates only slightly; this, added to the smoothing used in the statistical analysis, is the reason for the broadening seen in Fig. 8(b). No other narrowband features have been observed. The similarity of the ELM and $\mathrm{L}$ mode spectra gives additional confidence in the validity of the multiple interval averaging technique.

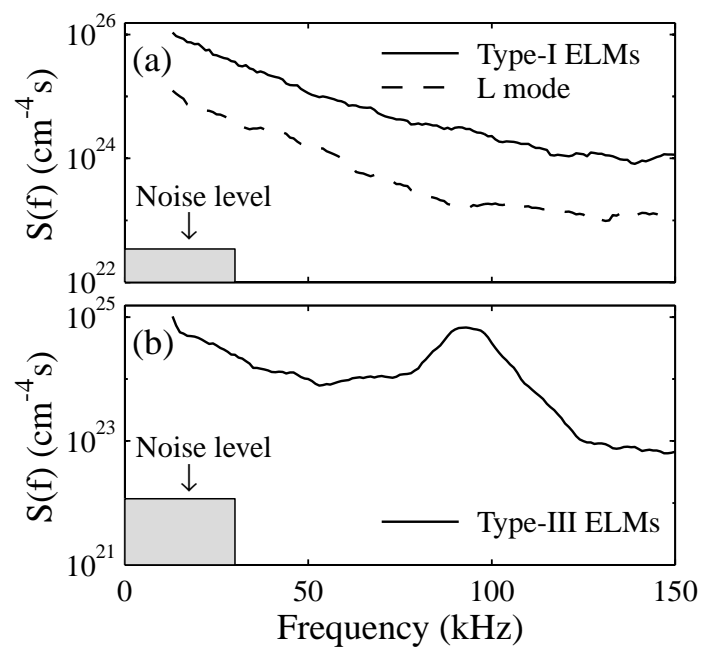

Figure 8. PCI autopower spectra shown on a logarithmic scale for two discharges: (a) comparison of type I ELMs (solid curve) and L mode (dashed curve); (b) type III ELMs. The spectra are estimated over (a) $10 \mathrm{~ms}$ and (b) $19 \mathrm{~ms}$ intervals with $10 \mathrm{kHz}$ smoothing, with a statistical uncertainty of (a) $10 \%$ and (b) $7 \%$; in the case of the ELMs, the intervals are composed of discontinuous $1 \mathrm{~ms}$ segments coinciding with individual ELMs. The location of the PCI chord is (a) $1 \mathrm{~cm}$ and (b) $1.7 \mathrm{~cm}$ inside the LCFS. Plasma parameters: (a) $B_{T}=2.16 \mathrm{~T}, I_{p}=2.0 \mathrm{MA}, \bar{n}_{e}=4.4 \times 10^{13} \mathrm{~cm}^{-3}$ (discharge 79641); (b) $B_{T}=2.1 \mathrm{~T}, I_{p}=1.0 \mathrm{MA}$, $\bar{n}_{e}=5.5 \times 10^{13} \mathrm{~cm}^{-3}$ (discharge 74455).

The ELM spectrum is found to have little spatial dependence, both in shape and in absolute value, across the range accessed by PCI, including locations deep in the SOL. Since the L mode fluctuation level is, by contrast, substantially lower in the deep SOL than in the main plasma [15], the ratio of the ELM level to the $\mathrm{L}$ mode level becomes generally quite large in the SOL.

\subsection{Wavenumber spectra and dispersion relations}

The same segmentation technique can be employed also to generate the complete frequency and wavenumber spectrum $S\left(k_{R}, f\right)$. Typical examples for type I and type III ELMs are shown in Fig. 9. Again, both types of spectra (with the exception of the high frequency coherent feature for the type III ELMs) are qualitatively similar to the L mode spectrum. In particular, both peak at nonzero wavenumbers, both positive and negative. This peculiar feature of this region of the spectrum $\left(k_{\theta} \sim\right.$ 0 ) is consistent with theoretical predictions for the 


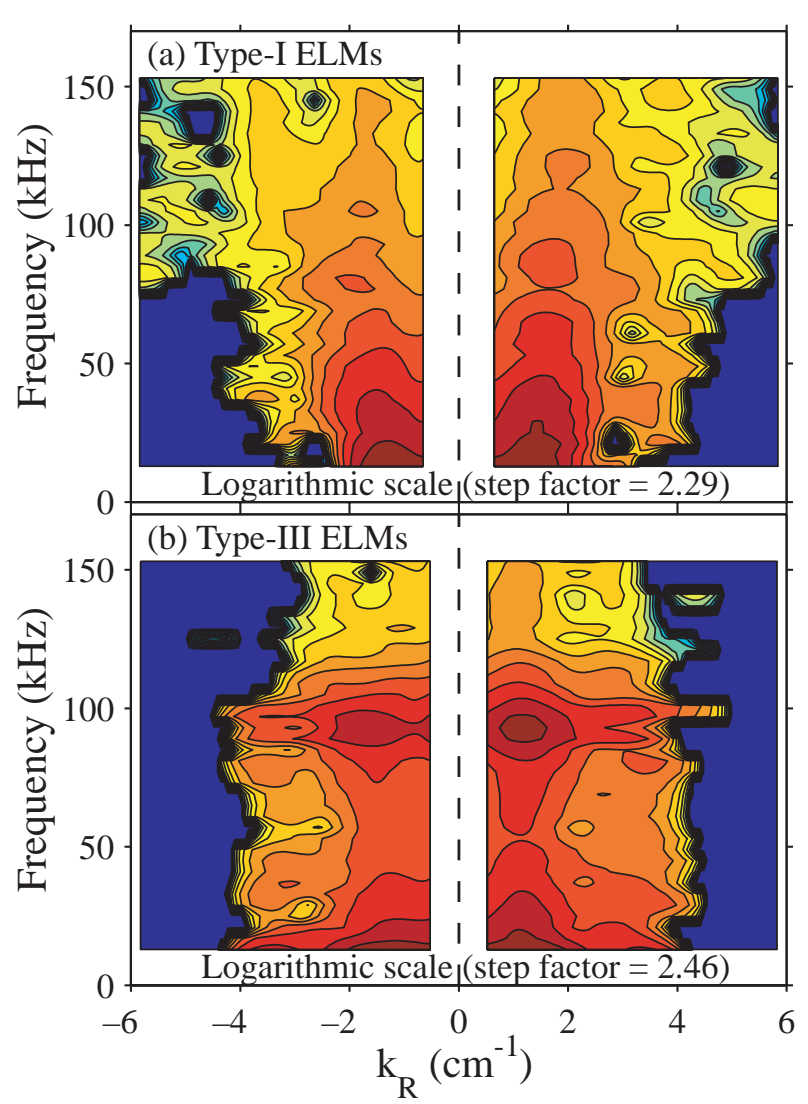

Figure 9. Absolute $S\left(k_{R}, f\right)$ spectra estimated over multiple (a) type I and (b) type III ELMs by averaging over (a) 10 and (b) 19 separate $1 \mathrm{~ms}$ intervals, in the region between the LCFS and (a) $3.5 \mathrm{~cm}$ inside and (b) $2.3 \mathrm{~cm}$ inside. Smoothing was performed over a $10 \mathrm{kHz}$ interval. The data used are the same as those for Fig. 8.

so-called radial modes [27] or zonal flows [28] that are believed to be the chief regulating mechanism for the overall turbulence level [22]. The main differences between the ELM and L mode spectra are a tendency of the ELM spectrum to peak at lower values of $k_{R}$ (typically in the neighbourhood of $1 \mathrm{~cm}^{-1}$ instead of $2-3 \mathrm{~cm}^{-1}$ ) and a slower increase of its peak wavenumber with frequency. In addition, no instances of predominantly inward propagating spectra have been found during ELMs: net propagation is always outwards, although it is nearly balanced in many cases (as in Fig. 9); by contrast, both inward and outward propagation have been observed in $\mathrm{L}$ mode [22].

These considerations can be visualized more readily by examining the three fitting parameters $k_{0, R}$, $L_{c R}$ and $P_{R}$ (cf. Eq. (1)), as functions of frequency. These functions are shown in Fig. 10 for the two

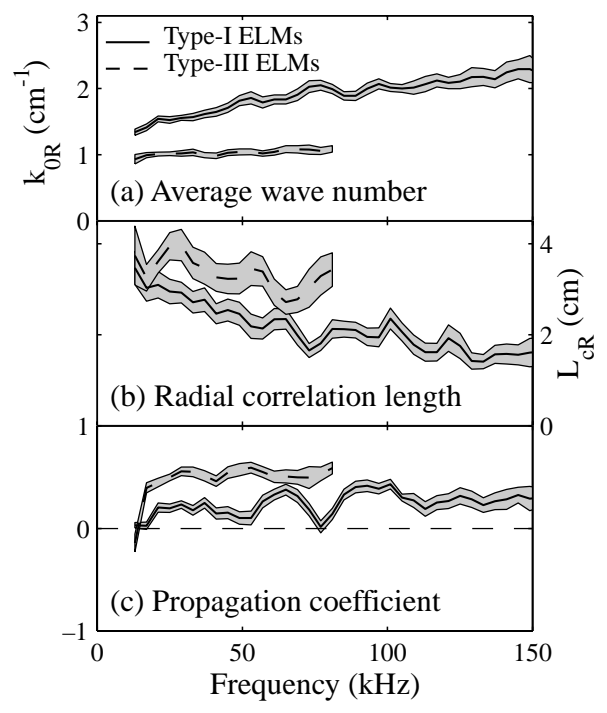

Figure 10. (a) Average radial wavenumber (dispersion relation), (b) radial correlation length and (c) propagation coefficient as functions of frequency, estimated over multiple ELMs by averaging over ten separate $1 \mathrm{~ms}$ intervals (type I ELMs, solid lines) and over 19 separate $1 \mathrm{~ms}$ intervals (type III ELMs, dashed lines) in the region between the LCFS and $3.5 \mathrm{~cm}$ inside (type I ELMs) or $2.3 \mathrm{~cm}$ inside (type III ELMs). The data used are the same as those for Figs 8 and 9 . The region above $80 \mathrm{kHz}$ is suppressed for type III ELMs as coherent activity invalidates the fits. Smoothing was performed over a $10 \mathrm{kHz}$ interval. The shaded areas indicate confidence intervals.

types of ELM (the type III ELM functions are truncated below the coherent mode, for which the fitting becomes meaningless). The dependence on frequency is weaker for type III than for type I ELM turbulence. Typical L mode dispersion relations display a more rapid increase of $k_{0, R}$ with frequency than the functions shown here. The behaviour of the correlation length for ELMs is qualitatively similar to the $\mathrm{L}$ mode case, but its absolute value is generally approximately a factor of $2-3$ larger. The propagation coefficient is always positive, confirming that net propagation is always outwards.

The coherence function between the PCI signals and the magnetic field fluctuation measured by Mirnov coils has been examined in a number of discharges. The coherence is effectively zero for the broadband turbulence, i.e. it never exceeds the minimum significant statistical value. This is not surprising, as the coherence level between the density fluctuations themselves becomes negligible beyond a distance of $4-5 \mathrm{~cm}$. Unfortunately no fast magnetic 


\section{S. Coda et al.}

data were available for the set of shots in which the type III ELM coherent mode was observed, rendering a cross-correlation analysis impossible for this case.

\section{Discussion and conclusions}

The broadband turbulence within a type I or type III ELM in the long poloidal wavelength region of the spectrum is, within the resolution of the measurements, similar to its L mode counterpart. In particular, the frequency spectra are entirely analogous; the characteristic radial spectral lengths (correlation length and average wavelength) are longer for ELMs, and their monotonically decreasing dependence on frequency is somewhat weaker. The main differences between ELM and L mode turbulence are in amplitude (typically a factor of 3-4 higher for ELMs), in spatial location (predominantly in the vicinity of the LCFS in L mode, whereas it encompasses most of the SOL during an ELM) and in the predominant radial direction of propagation (indifferent in L mode, always outward during an ELM).

In view of the similarity of the turbulence signatures in L mode and during an ELM, the ELM could be described as an $\mathrm{H}-\mathrm{L}$ 'back'-transition followed by an $\mathrm{L}-\mathrm{H}$ transition. This interpretation was proposed earlier specifically for type I ELMs on the grounds that the fluctuation levels, the edge plasma rotation, and the density and temperature profiles during the ELM closely resembled their L mode counterparts [12]. It was then speculated that the remaining differences in the turbulence, as well as the greater speed of the ELM termination in comparison with an $\mathrm{L}-\mathrm{H}$ transition, could be ascribed to the different pressure profiles in the two cases. A later investigation [29] found clear instances of $\mathrm{H}-\mathrm{L}-\mathrm{H}$ sequences in the second phase of the so-called 'compound' type I ELMs but led to the conclusion that the ELM itself was purely an MHD event. Indeed, the amplitude of the broadband magnetic turbulence measured near the divertor strike point is significantly larger in an ELM than in L mode. However, our measurements clearly indicate that during both type I and type III ELMs turbulent, broadband zonal flows are generated whose characteristics are very similar to those seen in L mode. A complete theory of the ELM phenomenon would thus have to account for the nonlinear generation of zonal flows by the driving MHD instability. There are already some theoretical indications that zonal flows can be generated by resistive ballooning instabilities [30,31] and may indeed be a general feature of tokamak turbulence.
Since no significant spatial variation in the signal level is observed across the full width of the PCI beam during an ELM, the ELM event affects a broader region than that occupied by the transport barrier in $\mathrm{H}$ mode. This is consistent with previous observations: the ELM is indeed 'edge localized', but less so than the $\mathrm{H}$ mode transport barrier; its effects can be observed over the outer $20 \%$ of the plasma [3].

The chief difference between type I and type III ELMs, in terms of their broadband turbulence content, is the timing of their appearance in relation to the peak in the $\mathrm{D}_{\alpha}$ signal: the peak in the fluctuation amplitude precedes the $\mathrm{D}_{\alpha}$ peak by $0.4-0.6 \mathrm{~ms}$ in the case of a type III ELM, whereas the fluctuation amplitude is near or at its maximum at the time of the $\mathrm{D}_{\alpha}$ peak for a type I ELM. The magnitude of the delay for type III ELMs is well in excess of the propagation time for a sound wave from midplane to divertor, while it is qualitatively consistent with typical growth times involved in resistive MHD instabilities [32]; it may then be argued that the onset of a resistive ballooning instability [31], believed to trigger the type III ELM [10], would cause transport and expulsion of particles on such a timescale, after which a peak in the recycling signal can be expected to occur. By contrast, ideal MHD timescales are in the microsecond range; this is consistent with the lack of measurable delay seen in the case of type I ELMs.

The broadband precursor activity found before type I ELMs using both PCI and reflectometry [12] is too long lived to be related to MHD timescales and would rather appear to be associated with the slow evolution of the edge pressure profile in $\mathrm{H}$ mode on a transport timescale. It should be noted that the reflectometry results documented precursors for up to $50 \mathrm{~ms}$ before an ELM, whereas they are never observed by PCI more than 2-3 ms before an ELM. It is possible, however, that the different spatial locations and spectral ranges of the two measurements may account for the discrepancies, which would then simply reflect different manifestations of the same phenomenon.

The peculiar echoes of type I ELMs in the SOL are difficult to interpret owing to their apparent randomness. It may be speculated that, as the rate of transport returns to $\mathrm{H}$ mode levels after the occurrence of an ELM, the readjustment of the profiles may lead to local violations of the ideal ballooning stability condition in the SOL.

An important question in any investigation of ELMs is the relation of the broadband turbulence 
to the various MHD instabilities that are believed to trigger the ELMs. It is generally believed that the onset of the MHD mode causes a self-consistent adjustment of the plasma equilibrium [33], which leads in turn to an increased level of turbulence, probably through the observed temporary slowing down of the sheared flows [34] that regulate turbulence in $\mathrm{H}$ mode. The ELM is terminated when the conditions for $\mathrm{H}$ mode are re-established.

This general picture is consistent with the lack of coherent activity observed by PCI during or before a type I ELM, again because of the rapid growth of ideal MHD instabilities (in the microsecond range); however, it should be noted that occasional bursting semicoherent precursor activity to type I ELMs has been documented by reflectometry in DIII-D [12], in possible disagreement with this interpretation.

The considerably slower growth rates of resistive MHD events may explain the observation of a coherent mode during the duration of a type III ELM (Fig. 5). This may also be a manifestation of a semicoherent mode found earlier by reflectometry measurements [35]: in that case, the precursor oscillations were observed to move radially outwards during a period of 10-20 ms until they triggered the ELM; at this point, they should indeed be expected to be visible on the PCI signal, which is only affected by the outermost region of the plasma.

\section{Acknowledgements}

The authors wish to thank the DIII-D Team for the operation of the tokamak, heating systems and diagnostics. Valuable discussions with P.H. Diamond are gratefully acknowledged. This work was supported by the US Department of Energy under Grant No. DE-FG02-91ER54109 and Contract No. DE-AC03-89ER51114.

\section{References}

[1] Keilhacker, M., et al., Plasma Phys. Control. Fusion 26 (1984) 49.

[2] Groebner, R.J., Phys. Fluids B 5 (1993) 2343.

[3] Zohm, H., Plasma Phys. Control. Fusion 38 (1996) 105.

[4] Hill, D.N., J. Nucl. Mater. 241-243 (1997) 182.

[5] Leonard, A.W., et al., J. Nucl. Mater. 266-269 (1999) 109.

[6] Connor, J.W., Plasma Phys. Control. Fusion 40 (1998) 191.

[7] Gohil, P., et al., Phys. Rev. Lett. 61 (1988) 1603.

[8] Ferron, J.R., et al., Phys. Plasmas 7 (2000) 1976.
[9] Osborne, T.H., et al., Plasma Phys. Control. Fusion 42 (2000) A175.

[10] Zohm, H., et al., Nucl. Fusion 32 (1992) 489.

[11] Ozeki, T., et al., Nucl. Fusion 30 (1990) 1425.

[12] Doyle, E.J., et al., Phys. Fluids B 3 (1991) 2300.

[13] Kass, T., et al., Nucl. Fusion 38 (1998) 111.

[14] Reimerdes, H., Pochelon, A., Suttrop, W., Nucl. Fusion 38 (1998) 319.

[15] Coda, S., Porkolab, M., Burrell, K.H., Phys. Lett. A 273 (2000) 125.

[16] Coda, S., Porkolab, M., Carlstrom, T.N., Rev. Sci. Instrum. 63 (1992) 4974.

[17] Coda, S., An Experimental Study of Turbulence by Phase-contrast Imaging in the DIII-D Tokamak, PhD Thesis, Massachusetts Institute of Technology (1997). http://www.psfc. mit.edu/library/rr/97rr013_abs.html (pdf) and http://theses.mit.edu:80/Dienst/UI/2.0/Describe/ 0018.mit.theses\%2f1997-322 (gif).

[18] Zernike, F., Physica 1 (1934) 689.

[19] Gohil, P., et al., Nucl. Fusion 34 (1994) 1057.

[20] Liewer, P.C., Nucl. Fusion 25 (1985) 543.

[21] Coda, S., Porkolab, M., Rev. Sci. Instrum. 66 (1995) 454.

[22] Coda, S., Porkolab, M., Burrell, K.H., Phys. Rev. Lett. 86 (2001) 4835.

[23] Coda, S., Porkolab, M., in Controlled Fusion and Plasma Physics (Proc. 21st Eur. Conf. Montpellier, 1994), Vol. 1, European Physical Society, Geneva (1994) 854.

[24] Bendat, J.S., Piersol, A.G., Random Data: Analysis and Measurement Procedures, Wiley, New York (1986).

[25] Censor, Y., SIAM Rev. 23 (1981) 444.

[26] Lazarus, E.A., et al., in Plasma Physics and Controlled Nuclear Fusion Research 1994 (Proc. 15th Int. Conf. Seville, 1994), Vol. 1, IAEA, Vienna (1995) 609.

[27] Waltz, R.E., Kerbel, G.D., Milovich, J., Phys. Plasmas 1 (1994) 2229

[28] Lin, Z., Hahm, T.S., Lee, W.W., Tang, W.M., White, R.B., Science 281 (1998) 1835.

[29] Zohm, H., et al., Nucl. Fusion 35 (1995) 543.

[30] Carreras, B.A., Lynch, V.E., Garcia, L., Phys. Fluids B 3 (1991) 1438.

[31] Rogers, B.N., Drake, J.F., Zeiler, A., Phys. Rev. Lett. 81 (1998) 4396.

[32] Rosenbluth, M.N., Rutherford, P.H., in Fusion, Vol. 1 (Teller, E., Ed.), Academic Press, New York (1981) 31.

[33] Lebedev, V.B., Diamond, P.H., Gruzinova, I., Carreras, B.A., Phys. Plasmas 2 (1995) 3345.

[34] Burrell, K.H., et al., Phys. Fluids B 2 (1990) 1405.

[35] Doyle, E.J., et al., in Controlled Fusion and Plasma Physics (Proc. 18th Eur. Conf. Berlin, 1991), Vol. 15C, Part I, European Physical Society, Geneva (1991) 285. 


\section{S. Coda et al.}

(Manuscript received 1 March 2001

Final manuscript accepted 20 June 2001)

E-mail address of S. Coda: Stefano.Coda@epfl.ch

Subject classification: D2, Te; D3, Te; I1, Te 